\title{
Profil d'évaluation du non-verbal dans la communication oralisée en public
}

\section{OpenEdition}

\section{Journals}

Édition électronique

URL : https://journals.openedition.org/communicationorganisation/2421

DOI : 10.4000/communicationorganisation.2421

ISSN : 1775-3546

\section{Éditeur}

Presses universitaires de Bordeaux

\section{Édition imprimée}

Date de publication : 1 novembre 2000

ISSN : 1168-5549

Référence électronique

Jacques Vermette, «Profil d'évaluation du non-verbal dans la communication oralisée en public », Communication et organisation [En ligne], 18 | 2000, mis en ligne le 27 mars 2012, consulté le 05 août 2021. URL : http://journals.openedition.org/communicationorganisation/2421 ; DOI : https://doi.org/ 10.4000/communicationorganisation.2421

Ce document a été généré automatiquement le 5 août 2021 .

(C) Presses universitaires de Bordeaux 


\section{Profil d'évaluation du non-verbal dans la communication oralisée en public}

Jacques Vermette

« Les insectes dépourvus d'intelligence composent du suc de différentes fleurs un miel dont toute l'industrie humaine ne saurait imiter

la saveur. Et nous nous étonnerons que

l'éloquence, ce don par excellence que la Providence a fait à l'homme, réclame l'assistance de plusieurs arts, qui, sans se manifester ouvertement dans le discours, lui communiquent cependant une force secrète, qui ne laisse pas de se faire sentir confusément!" Quintilien

1 À l'issue de mon mémoire de maitrise en psychopédagogie (1986) qui m'a permis de cerner l'aire de l'expression orale en public auprès d'une quarantaine de spécialistes, j'ai publié, en 1992, aux Presses de l'Université Laval : La parole en public. Dans ce livre, aux fins d'évaluation exhaustive de l'art oratoire quelle qu'en soit le genre (religieux, politique, pédagogique, judiciaire et social), j'ai déterminé une grille d'analyse en 6 paramètres - les attitudes, les pensées, le non-verbal, les mots, les sons et le matériel qui rassemble toutes les unités fondamentales d'une communication orale efficace.

2 Ln une telle grille d'analyse, j'ai établi, en trois paramètres chacun, deux axes structuraux. Dans le premier axe, la personnalité (les attitudes, le non-verbal et les sons) recueille 56 points sur 100 ; dans le deuxième axe, le contenu (les pensées, les mots et le matériel), compte 44 points.

Dans l'axe de la personnalité, le paramètre des attitudes (l'empathie, la confiance, l'authenticité et l'enthousiasme) totalise 28 points sur 56 ; le paramètre du non-verbal 
(la physionomie, la gestuelle, l'habillement et le maintien), 16 points ; le paramètre des sons (l'inflexion, le volume, l'articulation et le débit), 12.

Dans l'axe du contenu, le paramètre des pensées (l'intérêt, la clarté, la crédibilité et l'organisation) totalise 24 points sur 44 ; le paramètre des mots (l'adaptation, l'évocation, la justesse et la syntaxe), 12 ; le paramètre du matériel (la pertinence, la perceptibilité, l'esthétique et la maniabilité), 8. Lorsque l'orateur n'a pas eu recours à du matériel de logistique, les 8 points du matériel sont répartis entre les deux paramètres précédents : les pensées, 28 points ; les mots, ${ }^{16}$.

5 Les six paramètres, en leurs 4 éléments chacun, sont impliqués et imbriqués dans l'expression oratoire.

6 Mes pratiques professorales et professionnelles me confirment, en un ratio élevé, l'essentialiste du paramètre du non-verbal dans toute communication oralisée en public.

\section{Qu'est-ce donc que le non-verbal ?}

"On pourra posséder toute la puissance de l'intelligence, tout le feu d'imagination, tous les transports du cour, qui font la grande éloquence, si on n'a pas reçu de la nature les dons physiques correspondants, on ne sera qu'un orateur médiocre et impuissant." Jean Suberville

7 Le verbal est aux mots ; le non-verbal est « homo »...

8 Le non-verbal, c'est la face externe que, consciemment ou inconsciemment, le messager fait voir quand il parle de concepts et d'émotions.

9 Saviez-vous que votre corps peut envoyer jusqu'à 700000 signaux non verbaux ${ }^{1}$ ! le signal ne donne-t-il pas du corps à vos pensées?

10 Si jamais un conflit surgit entre l'expression non-verbale et les mots, Albert Mehrabian déclare que le public tend naturellement à croire le langage corporel $»^{2}$.

11 Le non-verbal, c'est l'esprit fait chair qui déploie sa stratégie pour capter l'attention, soutenir l'intérêt, faire comprendre et retenir à sa façon. Que l'orateur accorde donc sa chair à ce qui lui est cher !

12 Pour un orateur perspicace, quels sont donc les quatre éléments du non-verbal qui rendront sa communication orale efficace? Son expression non-verbale devra tirer profit du langage de sa « physionomie », de sa « gestuelle », de son « habillement » et de son « maintien. »

Pédagogiquement, abordons chacun des quatre éléments du non-verbal en des expressions décousues... mais, qui ont de la suite dans les idées. Dans les écoles, autrefois, les leçons ne nous étaient-elles pas inculquées à bâtons rompus?

14 Notez que l'approche humoristique de mes expressions ne se veut qu'une manière particulière pour vous mieux faire saisir mon message sans, toutefois, le griller.

"L'action oratoire, c'est l'éloquence du corps. C'est l'action qui domine dans l'art de la parole. » Cicéron. 


\section{La physionomie, c'est la transparence faciale du dit}

«Dans la communication, plus le leader politique est efficace sur le plan des mimiques et de sa gestuelle, moins le message verbal a d'importance. » René Zayan.

Faut, tôt, le savoir : vos apparences faciales vous copient l'âme en divers caractères.

Votre faciès, c'est votre univers, cité! Sur lui, campent les us et coutumes de votre savoir!

En toute évidence, par leur contractibilité, leur élasticité, leur excitabilité et leur tonicité, vos muscles faciaux mettent en mouvements, vos preuves neuronales : sinon, sur toute la ligne, c'est l'indice qui vous monte, en face, une farce!

Vos pensées verbalisées sont particulièrement expressives lorsqu'elles se montrent de face.

Pour un interlocuteur, vous voir de face si peu risquer votre peau, n'est-ce, pour lui, trop souvent payer cher votre expression en peau de chagrin!

En pleine face, les feux de la rampe mettent à jour même ce qui vous nuit !

En public, même par une belle façade, nous n'arrivons pas à sauver la face !

Votre face est la porte d'entrée du lieu où habite votre esprit : à grands traits, avec adresse, indiquez à vos spectateurs qu'ils sont à la bonne adresse.

Que la face éteinte de votre corps ne soit pas le corbillard de vos vives pensées!

À l'oral, n'est-il pas admiré celui qui monte au front? Visuellement, sans faux plis, il attaque de front sa pensée et nous la livre en ordre de bataille dont l'issue se perçoit bien à la manière fulgurante dont sont marqués ses traits cutanés. Sinon, à vous entendre, en visage flasque, les gens, assis sur leur cul..., deviendront « tannés »!

À bon teint, l'orateur qui bat les masques !

Une pensée qui. en mots, n'arrive pas à se mettre aux sons, laisse, en face, des traces : à cause d'un tel labeur, le visage est plein de micro-sillons...

Si l'orateur n'a pas visuellement d'appâts, il n'aura pas l'attention de son groupe : le public n'aime pas l'appât rance...

$\mathrm{Si}$, de fait, vous n'avez ni idées à soutenir, ni émotions à livrer, ni faits à rapporter, comment votre visage pourra-t-il envisager la transmission de preuves... sans perdre la face?

À votre public, livrez l'heure juste en pleine face; évitez les traits de la satire : c'est ainsi que la louange s'attire...

1 Ouvrez l'œil pour ne pas discourir aveuglément! Sinon, dans la salle, les fonds d'œil, aux iris ternes, vont se hérisser !

Quand vous parlez les yeux fermés, votre propos éclaire moins : l'auditoire se sent guidé à l'aveuglette !

Pour une réputation orale à la hausse, adressez, comme un preux, une parole relevée, les paupières dressées ; non pas comme un soldat aguerri qui combat la visière baissée ! À défaut, conspué, votre auditoire vous embaumera...

Savoir faire voir, telle est la clé de contact du démarrage des bougies d'allumage de la flamme oratoire qui garantira, d'un bon œil, le passage du feu de vos entrailles en plein cœur de l'âme de vos récepteurs. 
35 À divers moments de l'exposé, regardez chacun dans les yeux : tous doivent se sentir personnellement concernés : sinon, pour avoir adopté un profil bas, aux yeux cernés de vos interlocuteurs, vous constaterez que des ennuis se profileront en leur humeur aqueuse!

Parlant ou pensant d'un contenu dont le propos réfère à un souvenir, la kinesthésie a prouvé que d'une réalité :

vue ; un sujet oriente, instinctivement, son œil vers le haut, dans la direction droite ou gauche ;

entendue ; un sujet oriente, instinctivement, son œil vers le côté, dans la direction droite ou gauche ;

ressentie ; un sujet oriente, instinctivement, son œil vers le bas, dans la direction droite ou gauche ;

Il ne faudrait pas oralement n'évoquer que des souvenirs... en vous regardant, votre public n'apprécierait de votre œil que son globe trottant !

41 Quand vous regardez trop souvent le plancher, les mur ou le plafond, par un tel style terre à terre, les gens de votre auditoire se rendent vite compte que vous êtes en train de plafonner!

42 "Le nez de Pinocchio s'allonge lorsqu'il ment, celui des hommes gonfle. Selon une équipe de psychiatres de la Faculté de médecine de l'illinois, aux États-Unis, lorsqu'on ment, certains tissus enflent à l'intérieur du nez. Ce gonflement, baptisé "effet Pinocchio ", provoque une démangeaison qui conduit le menteur à se frotter l'appendice nasal. Les médecins se sont penchés sur les images de l'interrogatoire de Bill Clinton au cours du "Monica gâte ». Le président des États-Unis, qui n'était pas enrhumé, s'est frotté le nez toutes les quatre minutes. Pour les psychiatres, ses mensonges se voyaient donc comme le nez au milieu du visage». Philippe Chambron.

\section{La gestuelle porte, à bout de bras, la représentation signifiée du dit}

"Les mains portent en elles une intelligence des choses quotidiennes et savent des tendresses ou des labeurs qui $n$ 'ont pas de nom; elles ont aussi la capacité de dire ce pour quoi l'intellect $n$ 'a pas encore ou $n$ 'a plus de vocabulaire. Elles ne serrent que le vide; ce qu 'elles désignent pourtant $n$ 'est pas une absence, mais une aspiration ou une certitude de la foi.». Michel de Certeau.

Les interlocuteurs ne réfléchissent souvent qu'aux tours de bras qui fléchissent!

Que vos gestes qui se voient soient accompagnés de voix, pour qu'ils ne soient pas sans voie!

À vos gestes bien ciselés, unissez une pensée bien sculptée : distinguez-vous !

Vos gestes, il faut les prendre aux mots : ils doivent figurer ce que, à terme, vos termes expriment, parfois, d'indigeste.

À vos gestes nouveau-nés. accordez-leur un droit de passage pour que, dès leur enfantement, ils puissent, gracieusement, respirer un air de vérité : sinon, que d'avortons en dits gestes!

Pour une meilleure forme, n'oubliez pas que vos gestes ont parfois besoin de régime ! 
Quand les arabesques de vos gestes mettent vos propos en perspective, comme il se doit, les gens saisissent mieux le relief élégant de vos pensées.

Dans le déploiement de vos gestes, souciez-vous de l'air qu'ils ont pour que votre public soit ému du courant d'air qu'ils dégagent. A peine engagés, ne les étouffez pas en leur coupant le cou : laissez-les prendre l'air !

Par votre mode d'expression bien articulée en biceps, vous passerez pour un "fier à bras » : sinon, de votre public, en paumes, ne comptez pas sur un bras d'honneur !

C'est par les mains et les bras que les gestes sont exécutés ; sans eux, c'est l'orateur qui l'est par un public qui perd la tête!

Un geste ferme qui a de la classe est un geste habité qui ne déclasse pas l'orateur.

Un geste indéfini est un geste qui a perdu son gène : il nous gêne! Par une approche gestuelle mal équarrie, vous risquez d'être taillé en pièces, sans gène.

Un geste dégainé en crise de nerfs met le public sur la touche!

Les gestes qui tombent en masse ne sont pas de ceux qui enfoncent et rivent le mieux le clou de la connaissance.

Ln tenailles, n'est pas rivé à votre exposé l'interlocuteur qui, à tours de bras, se fait défoncer le nerf optique! Alors, n'attendez pas à demain, en mains, changez d'optique...

Pour être vus afin de créer leurs effets à la tonne, vos gestes projetés, pour ne pas que votre public en pleure, ont besoin d'ampleur: sinon, vos gestes détonneront, c'est clair...

9 De peur de recevoir des coups bas de la part de votre public, ne placez pas, en cachesexe, vos deux mains croisées : ainsi paumé, par votre sexe sous elles, vous passeriez pour un orateur pudique qui craint le tire-au-flanc!

Éloquemment, quand nos gestes sont au poil, c'est là qu'on signe de tout notre être !

Sous votre jupe, ne gardez pas captives vos mains : en sage-femme, osez les mettre au monde librement en coupant vous-même le cordon qui les retiennent collées à vos cuisses! Sinon, en vous envoyant promener, votre public mettra en l'air votre exposé " poigné»!

62 Au train où iront vos gestes, le public vous raillera en dormant ou cheminera avec vous au gré de vos envois musclés !

3 Nos gestes sont exhibitionnistes : à nu, sans s'en priver, ils affichent en public ce que notre esprit pense ! À défaut, ils font de l'embonpoint !

Un geste qui manque de retenue, c'est un geste qu'on laisse sans laisse!

Un geste qui ne parvient pas à destination est un geste qui manque d'adresse !

Les gestes, sans but fonctionnel, sont à exclure; ils ne passeraient que pour des spasmes musculaires...- ils ne serviraient donc qu'à vous aplatir, plutôt qu'à vous applaudir!

En public, les gestes les plus mal exécutés sont ceux que vous avez préalablement déterminés et que vous glissez sur votre parcours oratoire. Horodatés par antidate, manquant de synchronisme immédiat, ils ratent l'heure de tombée et, sur l'heure, heurtent, à contretemps, votre public mécontent.

"Sous la pulsion de notre inconscient, nos gestes sont porteurs de sens. Celui qui se frotte le nez, les cheveux ou les oreilles: ajouterait un démenti à ses propos; sinon, un embarras 
certain ci ce qu'il dit ou sa manière de le dire. Celui qui joint ses mains en forme de pyramide: dégagerait une attitude de supériorité. Celui qui se tape les cheveux: signifierait $q u$ 'il approuve ce qu'il énonce. Celui qui se frotte les mains: révélerait un plaisir certain à s'entendre. Celui qui se pince le nez: soulignerait la profondeur de sa réflexion introvertie. Celui qui nettoie ses verres, mord le bout de son crayon, regarde sa montre: soulignerait son désir d'interrompre l'échange au plus tôt. ». Ivor Davis.

\section{L'habillement, c'est l'apprêt étoffé du dit}

"Aux États-Unis, chez les gens d'affaires, l'habillement joue jusqu'à $84 \%$ dans le choix d'un candidat dans l'engagement à un poste de cadre. ». John Malloy.

Sur scène, habilement en votre habillement, brillez de mille feux !

Que le scintillant de votre paraitre révèle le lustre de votre être !

Soyez apparemment ajusté sur mesure : fond et forme brillamment couplés.

Assurez-vous que votre parure ait l'étoffe de votre âme.

Que ce qui vous revêt ne couvre pas la valeur que vous portez!

Pour intéresser par son paraître, il suffit d'être en son essence plus qu'en ses accidents.

Que votre paraître ne déguise pas votre être : n'allez pas vous affubler d'une approche vivement chargée quand votre intérieur est vide et terne !

Ce n'est pas en portant des vêtements haut de gamme que vous serez nécessairement respectueux de l'étiquette!

Quoique vous soyez dans une tenue quelque peu relevée, il ne vous faudrait pas pour autant que vous affichiez un air hautain.

N'oubliez pas que vos vêtements vous découpent une certaine réputation: les canons de la beauté peuvent compromettre ou ternir votre honneur !

Bien qu'il faille être propre, il n'est pas requis d'être en grande toilette en laquelle, curieusement, vous risqueriez d'être exposé, en salle, sans aisance !

so Que vos ensembles soient signes de votre aplomb intérieur : à votre auditoire, ils ne doivent pas donner de fil à retordre !

1 En bon diplomate, respectez le protocole qu'exigent le goût des gens présents : soyez d'occasion...

Ne cherchez pas à vous cacher derrière vos appâts : vous ne gagneriez rien à leurrer votre auditoire par un extérieur attrayant, porteur d'un intérieur déplaisant. À trop vous appâter, vous risqueriez de tout empâter! Plût à tous que vous les épatiez autrement!

83 Si votre approche est sobrement assortie, les gens ne souhaiteront pas, indignement, votre sortie.

84 Ce n'est pas de la valeur des tissus dont est issue votre crédibilité : oralement, avoir de l'étoffe provient davantage de la logique de la trame de vos pensées que du type de fils qui contourne le profil de votre gabarit.

Pour que vos auditeurs ne reniflent pas la senteur de vos efforts oratoires, que votre parfum soit de bonne odeur! Que votre flagrance n'entre pas en flagrant délit avec le bon goût flairé de tous ! 

clairement dessiner les linges qui couvrent les secrets révélés des spécificités que vous offrez en garantie! Sur vous, en mal de convergence, les gens focaliseraient davantage leurs yeux que leur esprit. Vos pensées risqueraient d'être emportées par une vague de fond. fichu! En public, sous les feux d'un l'éclairage ardent, sauf en parade de mode.. attention aux jupes sans jupons : à trop vouloir foncer à fond de train, certains tissages, à coup de griffes, laissent voir, en clair, le type de sous-vêtements que vous exhibez... jusqu'en ses dentelles aussi ténues soient-elles!

93 Ne mettez rien en poche qui, sur scène, en plus de vous épaissir, pourrait raisonner plus fort que vous.

94 En public, à défaut d'être ailleurs, parez le coup d'un meilleur tailleur! Sur scène, n'êtes-vous pas tenu de faire votre nid. plutôt que votre lit!

"Les cravates de couleurs vives et avec des motifs audacieux sont poulies extravertis; celles qui ont des rayures claires et foncées expriment une personnalité indécise. Les larges rayures sur fond sombre vont à ceux qui sont toujours heureux. Quant aux pessimistes, ils optent pour le schéma inverse. On trouve les cravates multicolores au cou des hommes mécontents de leur passé; les gagnants préfêrent les cravates claires à motifs foncés, alors que l'inverse séduit les êtres effacés. Les traditionalistes portent des cravates à motif cachemire, tandis que ceux qui vont de l'avant affectionnent les motifs géométriques sombres sur fond clair. ". Colin Randall.

\section{Le maintien, c'est l'ossature visuelle du dit}

"Le corps est une grande intelligence. ». Nietzsche.

Dans l'arène oratoire, un corps avachi ne produit pas d'effet bœuf!

Un bon port témoigne normalement d'un être droit. 
97 La flamme oratoire n'ossifie pas les muscles. Il faut s'y fier; la vivacité du cœur assouplit l'ossature pour lui conférer une charpente gracieuse qui vous rendra agréable à voir. Les gens diront : quel port ! Vous plairez à autrui...

Lors de manifestation sociale, c'est l'allure de votre corps qui déterminera l'aisance de votre nature!

Sur scène, respectez une distance physique de bon aloi : de façon non autorisée par chacun, ne franchissez pas la bulle personnelle des personnes du groupe. D'un bon mètre de distance, vous passerez en maître ! Pour le reste : omettre. Autant que possible, parlez debout plutôt qu'assis: votre projection sera plus dynamique, sinon vous n'émettriez que des propos rassis.

101 Tenir à livrer vos pensées, passées au feu de l'éloquence, aide à soutenir votre corps qui ne doit pas les éteindre par son allure non embrasée : sinon, en une approche fausse, les gens vont vous tenir pour un mort... en sursis!

102 Vos passions libérées parcourront vos muscles pour les tonifier; votre body-building sera un tonifiant pour ceux qui vous regarderont en chair et en os. Édifiés, ils vous voueront un culte dur ! À défaut, ils vous confesseront leur respect ramolli...

103 Certains orateurs pensent que le déplacement sur scène indique une vitalité utile de leur être débordant. Ce n'est pas une belle mobilité ! C'est déplacé : ces orateurs, agités sur toute la ligne, seraient mieux alités !

104 Comme émetteur, sur scène, en allant incessamment de gauche à droite, n'allez pas en courant alternativement court-circuiter l'attention soutenue de vos récepteurs choqués. Montrez que vous avez du potentiel : arrêtez de sauter; branchez-vous, si vous voulez que vos auditeurs restent en ligne! Si vous ne le voulez pas : vos auditeurs d'humeur maligne vont disjoncter!

105 Sur l'estrade, ne vous tenez pas incliné, vous passeriez pour un communicateur qui, pour assurer la propulsion de ses propos diffusés, serait mal ajusté sur sa rampe de lancement.

106 Selon la tenue de votre haleine, les gens tireront au clair que, visuellement, vous avez du plomb dans l'aine! Dès lors, rien n'épatera...

107 Le squelette de votre corps doit, nettement, correspondre à l'ossature de vos pensées et à la structure de vos phrases: exprimées en sons bien charpentés. Sinon, sans ces soutiens, en gorge, vous n'aurez pas d'allure!

108 Pour garantir la crédibilité de vos avancés oralement exprimée, que votre maintien ne soit pas offert en contre preuve !

Pensez que votre corps peut avoir de la gueule !

110 Durant toute la durée de votre exposé, toutes fausses notes corporelles entachent la sécurité des avancées de vos idées ou de vos émotions.

111 D'après la psychologie des formes, comme une certaine corpulence vous ferait paraître plus vieux, plus conservateur, dans votre tenue, n'accentuez pas ces traits. Sinon, vous serez cru de moins en moins! Consolez-vous cependant, par cette morphologie, vous dégagerez un abord plus sociable!

112 Si vous avez un corps plus athlétique, vous passerez pour plus mûr, plus grand, plus aventureux. Selon l'objet de votre entretien, vous aurez avantage à modifier certains aspects de votre exposition. Si vous êtes mince, vous passerez de soi pour plus 
ambitieux, plus nerveux, plus pessimiste, plus tranquille. Surveillez alors votre style à fleur de peau...

En annonce, votre corporel est une donnée de marketing prioritaire de votre valeur tant que vous n'aurez pas ouvert la bouche pour : la soutenir ; ou la sous ternir !

Mettez de l'ordre dans vos os pour qu'ils arrêtent vos flasques muscles de faire tourner votre exposé en queue de poisson!

En son apparence, votre posture ne doit pas signifier l'imposture : à moins que vous ne soyez un imposteur!

Quelles que soient vos formes naturelles, dans votre tenue, soyez sans écart aux normes convenues : que votre tête soit redressée, mais non altière; que vos épaules ne soient pas relevées, ni tombantes; que votre torse soit bombé sans crâner; que vos bras soient détendus: que vos genoux ne soient pas plies: que vos jambes reposent bien sur la plante de vos pieds. Sinon, les gens ne seront pas tenus de vous écouter!

"Il allait prendre un maintien trop élégant et des gestes trop civilisés pour l'état qu'on devait lui supposer $»$. Alfred de Vigny.

\section{Épilogue}

En art oratoire, le non-verbal nous parle de l'orateur :

Pile, sa face nous montre son avers ;

Sans fil à retordre, ses vêtements taillent ou entachent sa réputation ;

Avec prestance, son maintien soutient ou conteste sa valeur.

\section{PROCESSUS ÉVALUATIF ${ }^{3}$}

En pourcentage (\%) vous évaluez la performence par élement (ex EMPATHIE 100)r

En points (Prs) vous atrubuez les chiffres correspondants(ex EMPATHIE7.0Prs)

Les quatre élements additionnées(EMPATHIE,CONTATE, AUTENCITE ? ENTHOSIASME ${ }^{\circ}$ donnent sur 28 le sous total d'un paramètre(ex ATTITUDES du secteur PERSONNALITÉ28 :Prs

le calcul du tris parametre par secteur(ex ATTITUDE NOM VERBAL ET SONS ${ }^{\circ}$ vous donne la somme de l'ensemle du secteur(ex PERSONNALITÉ56/100)

L'addition des deux scteur s (ex PERSONNALITÉ+CONTENU ${ }^{\circ}$ constitue le pointage final (ew 100/100)Prs

PROFIL D’ÉVALUATION DE LA COMMUNICATION ORALISÉE EN PUBLIC ${ }^{4}$ 
LA VALEUR DE L'ETRE DU COMNUNICATEUR

La mesure de ba consideration estimant la nchesse du sujel offram

I ATtToms L'rxpression micholociou.

1 rapratul: L'évident souci de :'orateur de parler à, avec.

2 confasce La sécurité intéricure et extcricure de son,

devant chacun des auditeurs presents

3 se rexexm L'adhésion profonde de l'orateur vibrant sincerement a sim conienu

4 Exmousins: La vitalité passionnelle de lorateur branchéc

III NOS Ve.kBaL. L'EXPRrssion CORPORELIE

9 Prosionomite La iransparcice faciale des attitudes, en rappont

10 Gestruue La reprecentation significe des bras et des

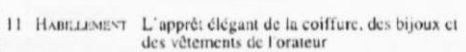

12 Mastier La posture cn contormité aux propos avancés

IV Sons L'Expkession sonoke.

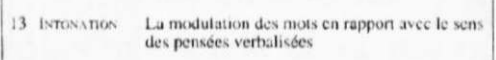

14 Voutu: Lintenste sonore de la projection des mots

I5 ARtrestatios La prononciation feme et netie des consonnes et ves syllabes des mots dits

16 Deur Le rythme de lélocation accordé au contenu

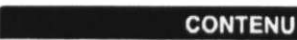

LA VALEUR DES IDÉES ET DES FMOTIONS DANS LA COMMUNICATION

La mesure de la considération cstimant la richesse de l' ebject of fer

II Pensers L'expression intela.ecto-affectivi

5 intiset Les bénéficess réels et profitables, issus de

6 Clarte Le démonstration nete des partics de tou:

7 CKedesultite La vérité dans les fatts, dans la logique des rai-

C

8

V Mots L'EXPRESSION GRAMULTICO-L.FICALE.

17 Anartation L'adequation du vocubulaire émis sclon ta

capacité compréhensible du recepteur

18 Evocatos L'apport des mots qui font vorr. cntendre.

19 Jusn sst L'emploi des noos justes pour traduire sus

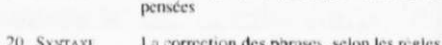

20 SvNTAXL. La correction des parases, selon les regles
grammaticales propres a la languc explotite

VI MatEkezL L'expressiox mimanouk.

21 Purtivesch. L'emploi de support de communication, par
nécessité ou utilité des recepteurs

22 PLrcismat.re La presentation aisée et for contrastes

pour bien traduire ses pensecs

23 Estwenout La projection agrcable, digne, harmonicuse c: simple des réalitís projetées

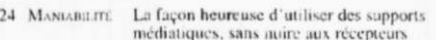

Nom

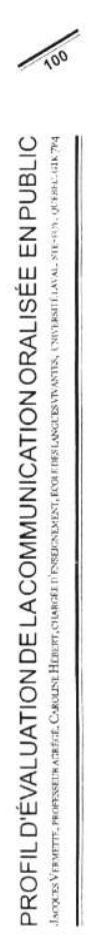

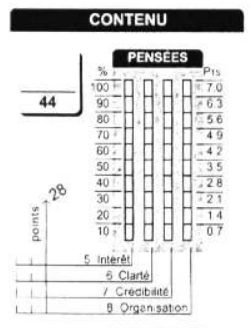

(1)
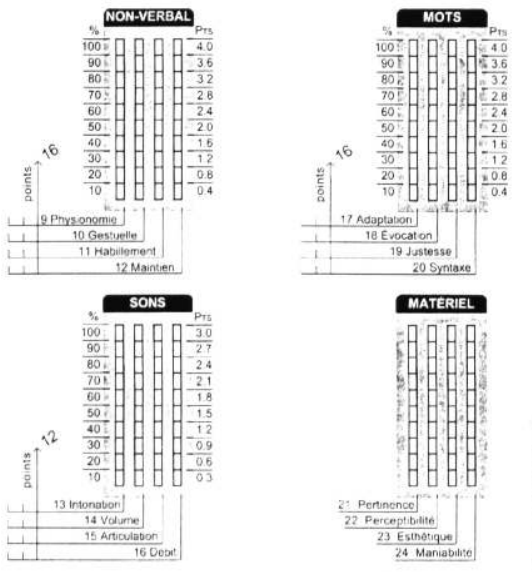


\section{NOTES}

1. Birdwhistell Roy L., Introduction to Kinesics, University of Louisville Press. 1952.

2. Mehrabian Albert, Non-verbal Communication, Chicago, Aldine-Atherton, 1972.

3. Dans ce texte, sans préjudice, le masculin inclut le feminin

4. Pour information, consultez le texte: Vermette Jacques, Cloutier Richard, «La parole en public », 1992. Presses de l'Université Laval, Sainte Foy, Quebec, Canada

\section{AUTEUR}

\section{JACQUES VERMETTE}

Université Laval, Québec 